OPEN ACCESS

Edited by:

Jason Roszik,

University of Texas MD Anderson

Cancer Center, United States

Reviewed by:

Duncan C. Gillbert,

Brighton and Sussex University

Hospitals NHS Trust,

United Kingdom

Luis De La Cruz-Merino,

Hospital Universitario Virgen

Macarena, Spain

*Correspondence:

Franz Rödel

franz.roedel@kgu.de

Specialty section:

This article was submitted to

Cancer Immunity and

Immunotherapy,

a section of the journal

Frontiers in Immunology

Received: 15 August 2017 Accepted: 15 September 2017 Published: 29 September 2017

Citation:

Martin D, Rödel F, Winkelmann R, Balermpas P, Rödel $C$ and Fokas $E$

(2017) Peripheral Leukocytosis is Inversely Correlated with Intratumoral

CD8+ T-Cell Infiltration and

Associated with Worse Outcome

after Chemoradiotherapy

in Anal Cancer.

Front. Immunol. 8:1225.

doi: 10.3389/fimmu.2017.01225

\section{Peripheral Leukocytosis Is Inversely Correlated with Intratumoral CD8+ T-Cell Infiltration and Associated with Worse Outcome after Chemoradiotherapy in Anal Cancer}

\author{
Daniel Martin', Franz Rödel'1,2,3*, Ria Winkelmann ${ }^{4}$, Panagiotis Balermpas ${ }^{1,2,3}$, \\ Claus Rödel ${ }^{1,2,3}$ and Emmanouil Fokas ${ }^{1,2,3}$ \\ ${ }^{1}$ Department of Radiotherapy and Oncology, Goethe University Frankfurt, Frankfurt, Germany, ${ }^{2}$ German Cancer Research \\ Center (DKFZ), Heidelberg, Germany, ${ }^{3}$ German Cancer Consortium (DKTK), Partner Site Frankfurt am Main, Frankfurt, \\ Germany, ${ }^{4}$ Senckenberg Institute for Pathology, Goethe University Frankfurt, Frankfurt, Germany
}

Peripheral blood leukocytosis has been implicated in promoting tumor progression leading to worse survival, but the mechanisms behind this phenomenon remain unexplored. Here, we examined the prognostic role of pretreatment white blood cell (WBC) count and clinicopathologic parameters in the context of CD8+ tumor-infiltrating lymphocytes (TIL) and myeloperoxidase+ tumor-associated neutrophils (TANs) in patients with anal squamous cell carcinoma (ASCC) treated with definitive chemoradiotherapy (CRT). After a median follow-up of 26 months, leukocytosis correlated with advanced T-stage $(p<0.001)$ and $\mathrm{N}$-stage $(p<0.001)$, and predicted for worse distant-metastasis-free survival $(p=0.006)$, disease-free-survival (DFS, $p=0.029)$, and overall survival $(p=0.013)$. Importantly, leukocytosis was associated with a lower intraepithelial CD8+ TIL density $(p=0.014)$, whereas low CD8+ TIL expression in the intraepithelial compartment was associated with worse DFS $(p=0.028)$. Additionally, high TAN expression in the peritumoral compartment was associated with a significantly lower density of CD8+ TIL ( $p=0.039$ ), albeit, TAN expression lacked prognostic value. In conclusion, leukocytosis constitutes an important prognostic marker in ASCC patients treated with CRT. In conjunction with intratumoral TIL and TAN, these data provide for the first time important insight on the correlation of peripheral blood leukocytosis with the intratumoral immune contexture and could be relevant for future patient stratification using immunotherapies in ASCC.

Keywords: anal cancer, leukocytosis, immune microenvironment, tumor-infiltrating-lymphocytes, neutrophils, CD8, myeloperoxidase

\section{INTRODUCTION}

Anal squamous cell carcinoma (ASCC) arises from the anal canal and is a rather rare malignancy with an incidence of two to three in 100,000 per year in the general population (1). While organ-preserving definitive chemoradiotherapy (CRT) remains standard of care, locoregional persistence or relapse still occurs in up to $30 \%$ of all patients $(2,3)$. Large randomized trials have already established T-stage, 
$\mathrm{N}$-stage, and gender as prognostic factors $(4,5)$. Nevertheless, due to the heterogeneity in treatment outcomes, there is still a need for reliable biomarkers to further stratify patients to treatment escalation or de-escalation.

The development of ASCC is associated with infection with high-risk strains of human papilloma virus (HPV) in 70-90\% of cases $(6,7)$. HPV positivity, as measured by immunohistochemistry or polymerase chain reaction, is associated with better treatment outcome after (chemo)radiotherapy $(7,8)$. Furthermore, there seems to be a link between HPV and immune contexture, as HPV positivity is associated with a more favorable tumor microenvironment (TME) (9).

The role of the immune TME in mediating cancer development and progression gained attraction in recent years. The positive prognostic impact of tumor-infiltrating lymphocytes (TILs) has been reported in several malignancies (10), including ASCC $(9,11-13)$. CD8+ T-cells have cytotoxic capabilities due to granules secreting perforin and granzyme $\mathrm{B}$, which are released upon detection of antigens via the major histocompatibility complex I and T-cell receptor (TCR) (14). In contrast, myeloid cells like neutrophils remain unexplored in ASCC. Neutrophils aid tumor progression and resistance to conventional anticancer treatments by mediating angiogenesis and suppression of CD8+ TIL-mediated antitumor immunity via secretion of inhibitory factors, e.g., inducible nitric oxide synthase (iNOS), arginase 1 (ARG1), and transforming-growthfactor beta (TGF $\beta)(15-17)$.

The negative impact of elevated pretreatment white blood cell (WBC) count in blood samples has already been reported in cervical and colorectal cancer (18-20). In ASCC, the same effect was described in recent retrospective studies (21-23). Leukocytosis was also correlated with adverse cancer-specific survival and overall survival (OS) in the ACT-I clinical trial (24). Leukocytosis in solid tumors can be caused directly by tumor cells via secretion of stimulatory factors such as granulocyte-colony-stimulating factor (G-CSF), granulocyte-macrophage-colony-stimulating factor, and upregulation of C-X-C chemokine receptor 2 (CXCR2) ligands (17). Nevertheless, no explanation for this observation has been provided so far.

Platelets play an important role in cancer progression. Clinical and preclinical evidence suggests that platelets can help tumor cells resist apoptosis, induce angiogenesis, facilitate chemoresistance, and help immune evasion during generation of metastasis $(25,26)$. Elevated pretreatment platelets are associated with worse outcome in colorectal cancer patients (27) and a higher propensity for metastasis (28).

In this study, we provide insight on the worse clinical outcome in patients with leukocytosis in correlation CD8+ TILs and tumor-associated neutrophils (TAN) in ASCC.

\section{MATERIALS AND METHODS}

\section{Patients and Treatment Protocol}

We identified 79 patients with newly diagnosed ASCC with available pretreatment formalin-fixed paraffin embedded (FFPE) tissues at our department. All patients were treated between 03/2003 and 05/2016 with definitive CRT and were routinely subjected to physical and rectal-digital examination, proctoscopy with biopsy, CT/MRI of the abdomen and pelvis, chest X-ray, serum chemistry, and complete blood count before initiation of therapy. Patients were staged according to UICC Version 8. Analysis was done following an institutional review board approval.

Chemotherapy was applied in the first and fifth week of radiotherapy (RT) and consisted of 5-fluorouracil (1,000 or 800 $\mathrm{mg} / \mathrm{m}^{2} /$ day) as 4 - or 5-day continuous infusion and Mitomycin $\mathrm{C}$ given as an intravenous bolus $\left(10 \mathrm{mg} / \mathrm{m}^{2}\right)$ on day one of each cycle. RT was applied using either 3D-conformal RT or intensitymodulated-RT (IMRT). Patients were treated with median total dose of 59.4 Gy (range, 34.2-64.8) with daily fractions of 1.8 or 2 Gy. 69 patients were treated with a median external boost dose of 9 Gy (range, 3.6-19.8).

\section{Follow-up}

Initial assessment of patients was done 8-10 weeks after completion of therapy and, afterward, every 3 months for the first 2 years followed by 6-month intervals. Examination on followup consisted of digital rectal examination, proctoscopy (with biopsies in case of suspicious lesions), and pelvic CT/MRI scan. No detectable tumor at first follow-up was defined as complete response (CR).

\section{Immunohistochemistry}

Immunohistochemical staining of myeloperoxidase (MPO) and CD8 was performed by a horseradish-peroxidase technique using a DAKO Autostainer Link 48 (DAKO, Hamburg, Germany) with standardized Dako EnVision ${ }^{\text {TM }}$ FLEX Blocking reagent (K800, DAKO) and polyclonal MPO antibody MPO

TABLE 1 | Patients, tumor, and blood characteristics.

\begin{tabular}{lcc}
\hline & & Median (range) or $\boldsymbol{n}$ (\%) \\
\hline Age, years & Male & $58(36-84)$ \\
Sex & Female & $34(43)$ \\
T-stage & T1 & $45(57)$ \\
& T2 & $22(28)$ \\
& T3 & $35(44)$ \\
N-stage & T4 & $16(20)$ \\
& N0 & $6(8)$ \\
Grading & N1 & $49(62)$ \\
& G1 & $30(38)$ \\
& G2 & $4(5)$ \\
& G3 & $50(63)$ \\
& Gx & $21(27)$
\end{tabular}

Pretreatment blood

counts

Leukocytes (/nl)

Platelets (/nl)

Hemoglobin (mg/dl)

Radiotherapy (RT)

RT modality

Total dose (Gy)

Boost dose (Gy)
$7.69(3.2-13.38)$

$266(111-531)$

$13.5(10.2-16.2)$

$\begin{array}{cc}\text { 3D conformal RT } & 32 \\ \text { Intensity-modulated-RT } & 47 \\ & 59.4(34.2-64.8) \\ & 9(0-19.8)\end{array}$


(dilution 1:1,000; A0398, DAKO) and CD8 (1:100, clone C8/144B; DAKO M7103). Next, dextran polymer conjugated horseradish-peroxidase and 3,3'-diaminobenzidine chromogen was used for visualization and hematoxylin solution (Gill 3, Sigma-Aldrich, Munich, Germany) for counterstaining. Negative control slides in the absence of primary antibodies were included for each staining.

The scoring of expression of MPO and CD8+ TIL was done semiquantitatively as described before (9). Categories for scoring were: (1) no, or sporadic cells; (2) moderate number of cells; (3) abundant occurrence of cells; (4) highly abundant occurrence of cells was done for both, intraepithelial, and stromal compartments ( $\times 10$ magnification) separately, and a total score was generated by addition of both scores as previously described $(29,30)$. We used the median value as cutoff for both compartments and the total score to divide the patient cohort in patients either having a low or high score. Images were acquired with the AxioImager Z1 microscope using the Axiovision 4.6 software (Zeiss, Germany). To minimize interobserver variability, two investigators (Daniel Martin and Emmanouil Fokas) without knowledge of the clinicopathologic data performed scoring. In cases of discrepancy, a final decision was made after additional examination of the specimens.

\section{Peripheral Blood Counts}

We derived the following baseline WBC counts and platelet counts from our hospital database. Differential blood counts were not available for a large proportion of patients. Baseline was defined as either the morning of treatment initiation or up to 4 days before this date. Leukocytosis was defined as having a WBC count equal or above the median. Additional analysis was done using a traditional cutoff of $10 / \mathrm{nl}$.

\section{Statistical Analysis}

Differences between groups were assessed using Pearson's Chisquared test for categorical variables and using the non-parametric Kruskal-Wallis test for continuous variables. Correlations were assessed using the Spearman's rank correlation coefficient. Survival times were calculated from start of CRT to the dates of respective events or last follow-up. Cumulative incidence of local failure was assessed using non-complete response at first restaging or locoregional recurrence after initial complete response as event. Disease-free survival (DFS) was calculated using the date of diagnosis of locoregional failure, distant metastases, or death of any cause. OS was calculated with death of any cause as the respective event. Remaining patients were censored for the respective end point. Survival curves were plotted using Kaplan-Meier plots and differences between curves were analyzed using the log-rank test. The Cox proportional hazard model was used for univariate analysis in order to assess the influence of WBC count and platelet count as a continuous variable. The assumption of proportional hazard was tested by assessing the scaled Schoenfeld residuals. Due to the low number of events, we were not able to conduct a multivariate analysis without risking overfitting (31). Statistical analysis was performed using $\mathrm{R}$ (Version 3.3) (32). A p-value of $<0.05$ was considered significant.

\section{RESULTS}

\section{Patient and Treatment Characteristics}

Patient and treatment characteristics are summarized in Table $\mathbf{1 .}$ Advanced T-stage $(p<0.001)$ and N-stage $(p<0.001)$ were significantly associated with an elevated WBC count (Figures 1A,B). There was a significant positive correlation between WBC count and platelet count $(r=0.59, p<0.001)$, whereas there was no significant difference according to gender, grading, and age.

\section{Leukocytosis and Intratumoral Immune Contexture}

The results of CD8 and MPO staining in pretreatment biopsies and correlations with clinicopathologic parameters are summarized in Table 2 and Table S1 in Supplementary Material. Male patients had a significantly higher intratumoral MPO+ TAN density compared to females $(p=0.03)$. There was no association between tumor size, nodal status, age, CD8+ TIL, or MPO+ TAN densities regardless of compartment. High TAN expression was associated with a significantly lower density of CD8+ TIL in the peritumoral (stromal) areas $(p=0.029)$. The same significant association also existed between peritumoral MPO+ TAN and CD8+ peritumoral TIL $(p=0.039)$. Representative images of low and high intratumoral and peritumoral CD8+ TIL and MPO+ TAN expression are shown in Figures 2A,B.
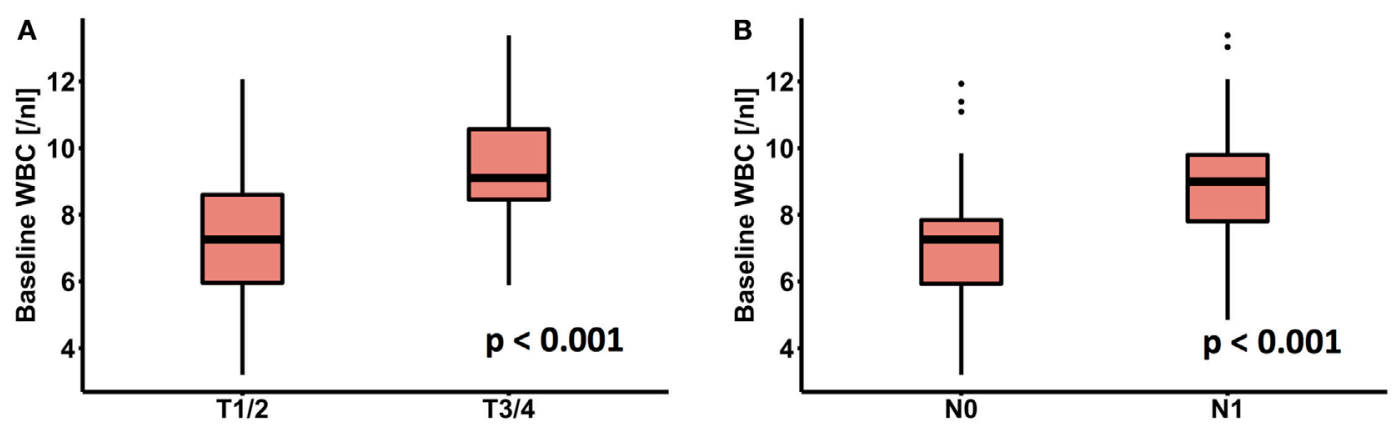

FIGURE 1 | Association of peripheral white blood cell (WBC) with (A) T-stage and (B) N-stage. 
TABLE 2 | Correlation of clinicopathological parameters with MPO and CD8 score according to different tumor compartments.

\begin{tabular}{|c|c|c|c|c|c|c|c|c|c|c|c|c|c|c|c|c|c|c|}
\hline \multirow[t]{2}{*}{ Parameter } & \multicolumn{3}{|c|}{ MPO stroma } & \multicolumn{3}{|c|}{ MPO tumor } & \multicolumn{3}{|c|}{ MPO total } & \multicolumn{3}{|c|}{ CD8 stroma } & \multicolumn{3}{|c|}{ CD8 tumor } & \multicolumn{3}{|c|}{ CD8 total } \\
\hline & Low, $n$ (\%) & High, $n(\%)$ & $p$-Value & Low, $n(\%)$ & High, $n(\%)$ & $p$-Value & Low, $n(\%)$ & High, $n(\%)$ & $p$-Value & Low, $n(\%)$ & High, $n(\%)$ & $p$-Value & Low, $n(\%)$ & High, $n(\%)$ & $p$-Value & Low, $n$ (\%) & High, $n(\%)$ & $p$-Value \\
\hline \multicolumn{19}{|l|}{ T-stage } \\
\hline$T 1 / 2$ & $17(68)$ & $40(74)$ & 0.57 & $47(69)$ & 10 (91) & 0.13 & $17(71)$ & 40 (73) & 0.86 & $48(70)$ & $9(90)$ & 0.18 & $36(67)$ & $21(84)$ & 0.11 & $29(66)$ & $28(80)$ & 0.17 \\
\hline $\mathrm{T} 3 / 4$ & 8 (32) & $14(26)$ & & $21(31)$ & $1(9)$ & & 7 (29) & $15(27)$ & & $21(30)$ & $1(10)$ & & 18 (33) & $4(16)$ & & $15(34)$ & $7(20)$ & \\
\hline \multicolumn{19}{|l|}{$\mathrm{N}$-stage } \\
\hline NO & $15(60)$ & 34 (63) & 0.8 & $42(62)$ & $7(64)$ & 0.9 & $14(58)$ & $35(64)$ & 0.65 & $41(59)$ & $8(80)$ & 0.21 & $30(56)$ & $19(76)$ & 0.08 & 24 (55) & 25 (71) & 0.12 \\
\hline N1 & $10(40)$ & $20(37)$ & & $26(38)$ & $4(36)$ & & $10(42)$ & $20(36)$ & & $28(41)$ & $2(20)$ & & $24(44)$ & $6(24)$ & & $20(45)$ & $10(29)$ & \\
\hline \multicolumn{19}{|l|}{ G } \\
\hline $\mathrm{G} 1 / 2$ & $15(60)$ & $38(75)$ & 0.2 & $44(67)$ & $9(90)$ & 0.13 & $14(58)$ & $39(75)$ & 0.14 & $46(70)$ & $7(70)$ & 0.98 & $33(65)$ & $20(80)$ & 0.17 & $26(63)$ & $27(77)$ & 0.19 \\
\hline G3 & $10(40)$ & $13(25)$ & & 22 (33) & 1 (10) & & $10(42)$ & $13(25)$ & & $20(30)$ & $3(30)$ & & $18(35)$ & $5(20)$ & & $15(37)$ & $8(23)$ & \\
\hline \multicolumn{19}{|l|}{ Gender } \\
\hline Male & $10(40)$ & $24(44)$ & 0.7 & $26(38)$ & $8(73)$ & 0.03 & $9(38)$ & $25(45)$ & 0.51 & $32(46)$ & $2(20)$ & 0.12 & $23(43)$ & $11(44)$ & 0.91 & $20(45)$ & $14(40)$ & 0.63 \\
\hline Female & $15(60)$ & $30(56)$ & & $42(62)$ & $3(27)$ & & $15(62)$ & $30(55)$ & & $37(54)$ & $8(8)$ & & $31(57)$ & $14(56)$ & & $24(55)$ & $21(60)$ & \\
\hline \multicolumn{19}{|l|}{ Age (years) } \\
\hline$<58$ & $13(52)$ & 25 (46) & 0.64 & $30(44)$ & $8(73)$ & 0.08 & $12(50)$ & $26(47)$ & 0.82 & $34(49)$ & $4(40)$ & 0.58 & $26(48)$ & $12(48)$ & 0.99 & $23(52)$ & $15(43)$ & 0.4 \\
\hline$\geq 58$ & $12(48)$ & $29(54)$ & & $38(56)$ & $3(7)$ & & $12(50)$ & 29 (53) & & $35(51)$ & $6(60)$ & & $28(52)$ & $13(52)$ & & $21(48)$ & $20(57)$ & \\
\hline \multicolumn{19}{|c|}{ CD8 stroma } \\
\hline Low & $19(76)$ & 50 (93) & 0.039 & $58(85)$ & $11(100)$ & 0.18 & $18(75)$ & $51(93)$ & 0.029 & & & - & & & - & & & - \\
\hline High & $6(24)$ & $4(7)$ & & $10(15)$ & $0(0)$ & & $6(25)$ & $4(7)$ & & & & & & & & & & \\
\hline \multicolumn{19}{|l|}{ CD8 tumor } \\
\hline Low & $16(64)$ & $38(70)$ & 0.57 & $46(68)$ & $8(73)$ & 0.74 & $15(63)$ & $39(71)$ & 0.46 & & & - & & & - & & & - \\
\hline High & $9(36)$ & $16(30)$ & & 22 (32) & $3(27)$ & & $9(37)$ & $16(29)$ & & & & & & & & & & \\
\hline \multicolumn{19}{|l|}{ CD8 total } \\
\hline Low & $12(48)$ & $32(59)$ & 0.3 .5 & $37(54)$ & $7(64)$ & 0.57 & $11(46)$ & $33(60)$ & 0.24 & & & - & & & - & & & - \\
\hline High & $13(52)$ & $22(41)$ & & $31(46)$ & $4(36)$ & & $13(54)$ & $22(40)$ & & & & & & & & & & \\
\hline
\end{tabular}

MPO, myeloperoxidase.

Significant values have been marked with bold. 

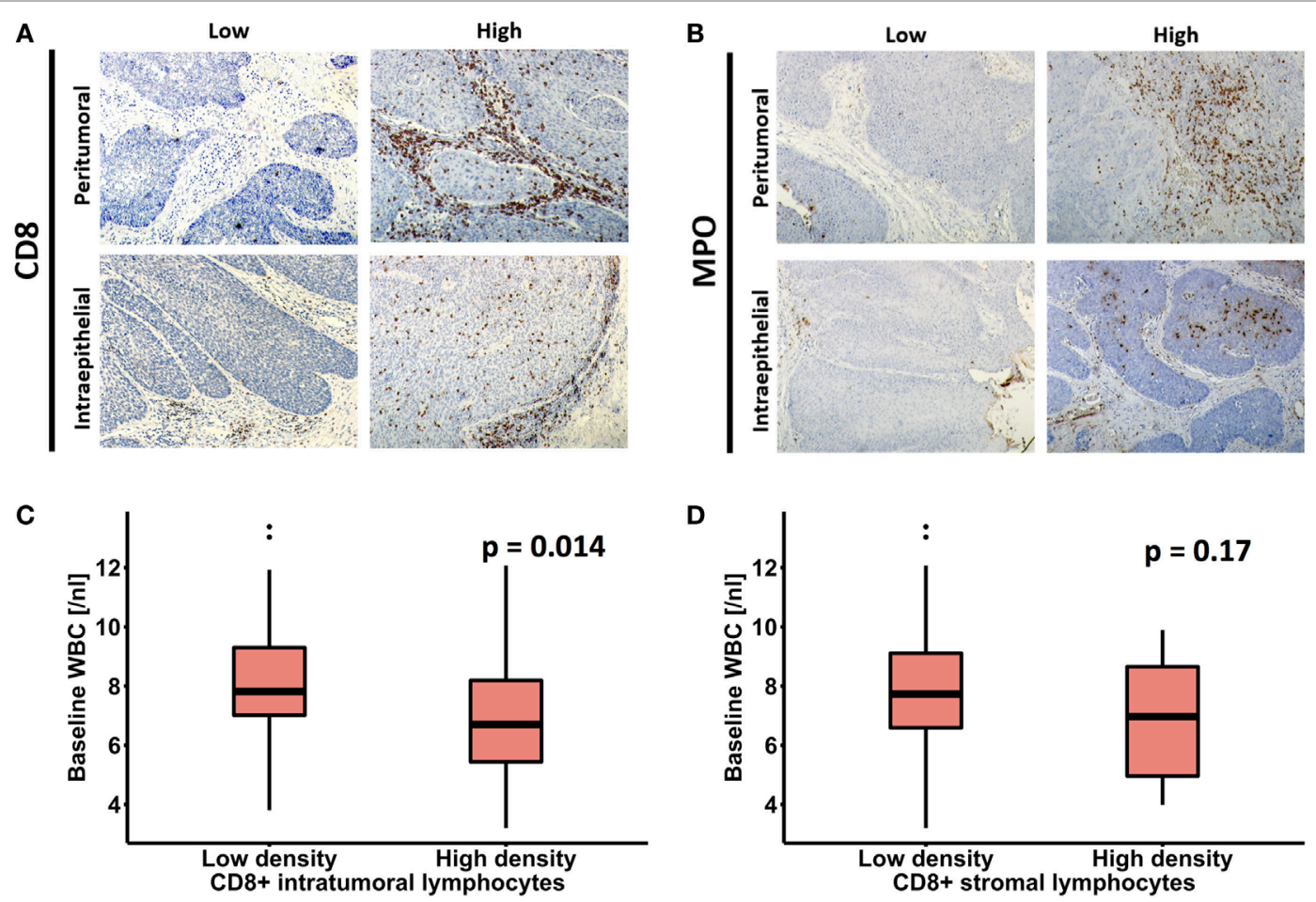

FIGURE 2 | (A,B) Example of immunohistochemical images showing high and low CD8 and myeloperoxidase (MPO) expression in pretreatment biopsies of patients with anal squamous cell carcinoma. Association of peripheral white blood cell (WBC) count with (C) intratumoral and (D) peritumoral (stromal) CD8+ T-cells.

Regarding the correlation of peripheral WBC and TME, pretreatment tumor specimens with a high intraepithelial CD8+ TIL expression had a significantly lower baseline WBC count $(p=0.014)$ compared to patients with no or only low infiltration, whereas there was no significant correlation between WBC and peritumoral (stromal) CD8+ TIL $(p=0.17)$ (Figures 2C,D). Similarly, we failed to detect a significant association between tumor MPO expression and peripheral WBC. Of note, patients with a high intraepithelial infiltration with CD8+ TIL had lower baseline platelets (Figure S1 in Supplementary Material).

\section{Treatment Outcome}

The median follow-up time was 26 months (range, 0-158). One patient died during therapy and $62(78 \%)$ patients had a complete response (CR) after CRT. Locoregional failure after initial CR occurred in $10(16 \%)$ patients, whereas 7 (9\%) patients were diagnosed with distant metastases. Furthermore, 12 (15\%) patients died during follow-up; $6(8 \%)$ due to ASCC, 2 (3\%) due to treatment-related complications, and $4(5 \%)$ due to unrelated disease.

Patients with a pretreatment leukocytosis ( $\geq$ median WBC count) had a significantly worse distant-metastasis-free survival (DMFS) $(p=0.0063)$, DFS $(p=0.029)$, and OS $(p=0.013)$ (Figures 3A-D). In an additional analysis that used WBC $\geq 10 / \mathrm{nl}$ as a cutoff as previously described $(21,23)$, leukocytosis was seen in $10(13 \%)$ patients and was associated with a significantly worse local control $(p=0.048)$, DMFS $(p=0.0021)$, DFS $(p=0.011)$, and OS ( $p=0.0067)$ (Figure S2 in Supplementary Material). To evaluate WBC count as a continuous variable, we used a cox proportional hazard model. Increase of WBC count was significantly associated with worse DMFS (HR 1.76; CI 1.22-2.55; $p=0.003$ ), DFS (HR 1.31; CI 1.06-1.62; $p=0.014$ ), and OS (HR 1.43; CI $1.09-1.86 ; p=0.009)$.

Regarding the impact of pretreatment platelet count, we found no significant prognostic impact using several cutoffs for dichotomization. In an additional univariate cox regression analysis using platelet count as a continuous variable, however, elevated platelet count was associated with worse DMFS (HR 1.009, 95\% CI 1-1.018, $p=0.048$ ).

A higher density of intratumoral CD8+ TIL was associated with a better DMFS $(p=0.049)$ and DFS $(p=0.028)$ (Figures 4A-D). There was no impact of the total score or the peritumoral density of CD8+ TIL on treatment outcome. Similarly, MPO+ neutrophil expression did not affect prognosis in our series.

\section{DISCUSSION}

In the present study, we aimed to investigate the clinical impact of peripheral blood leukocytosis in the context of intratumoral immune profile. Patients with ASCC that have baseline leukocytosis presented a worse outcome after CRT in line to previous observation in $\operatorname{ASCC}(21,23)$ and cervical cancer $(18,20)$. Our data confirm these results using the established cutoff point of 


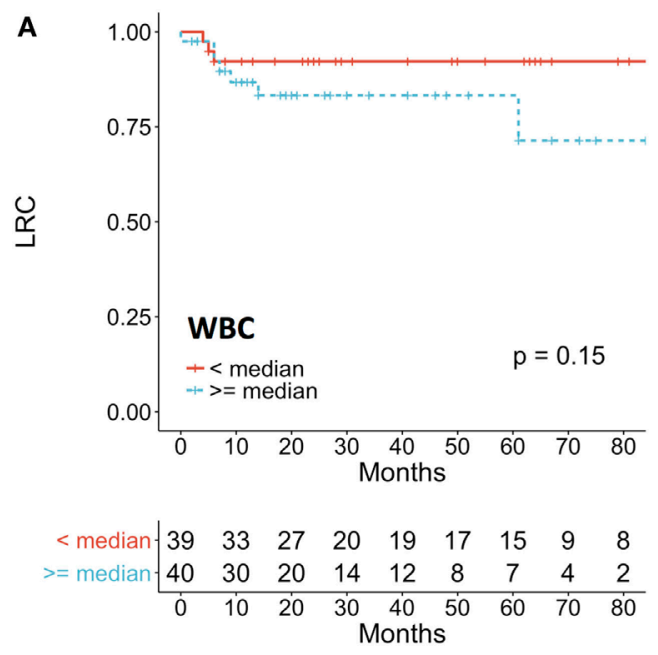

C

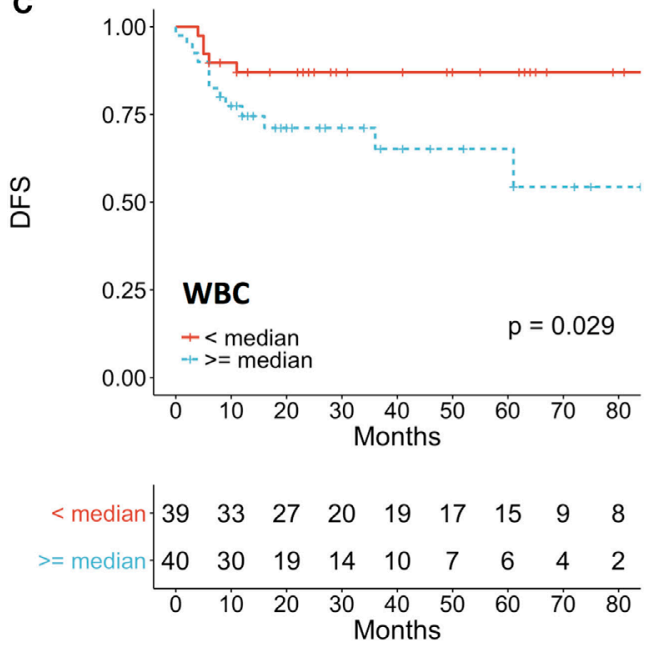

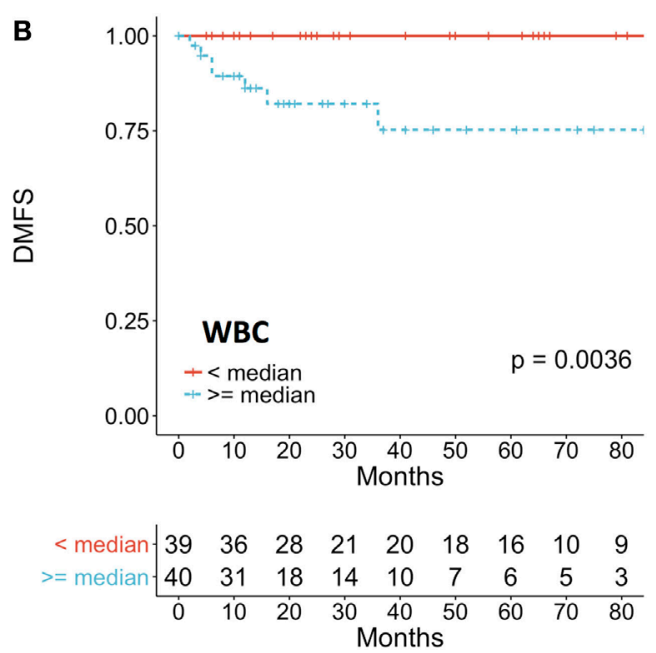

D

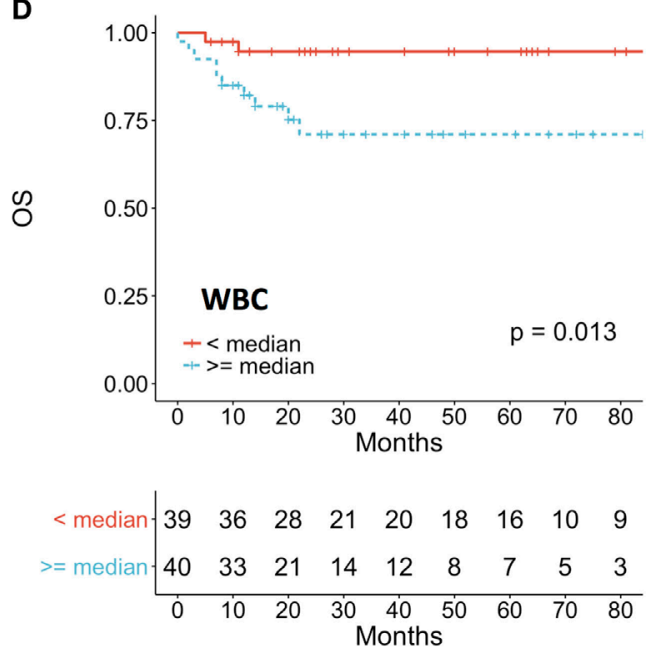

FIGURE 3 | Prognostic impact of white blood cell (WBC) count on (A) LRC, (B) DMFS, (C) DFS, (D) OS. Patients were dichotomized according to the median value of WBC count. LRC, locoregional control; DMFS, distant-metastasis-free survival; DFS, disease-free survival; OS, overall survival.

10/nl, but we found worse DFS and DMFS also in patients with moderately elevated WBC, based on median cutoff value.

In our work, patients with leukocytosis had a significantly lower intratumoral CD8+ TIL expression and vice versa. Leukocytes are important in acute inflammation to eliminate pathogens but, in a chronically activated state, they can promote tumor progression by secretion of TGF $\beta$, epidermal-growthfactor, and proteolytic enzymes (33). Importantly, leukocytosis as part of chronic inflammation can lead to inhibition of CD8+ T-cells via upregulation of programmed death 1 on T-cells and myeloid cells (34) that is in line to our findings. To the best of our knowledge, this phenomenon has not been reported yet and it proposes an additional explanation for the adverse role of leukocytosis in malignancies.

The role of TAN in ASCC has not been previously investigated. Notably, we found an inverse correlation between peritumoral TAN and CD8+ TIL densities. Several mechanisms have been implicated in the immunosuppressive effect of TAN. First, secretion of ARG1 lowers the expression of the $\zeta$ CD3 chain of the TCR leading to reduced activity $(35,36)$. Second, nitration of CC-chemokine ligand 2 by neutrophil-derived reactive nitrogen species reduces T-cell infiltration (37). Third, iNOS released by neutrophils inhibits proliferation of CD8 lymphocytes (16). Fourth, TGF $\beta 1$ signaling downregulates interferon gamma (IFN $\gamma$ ) in CD8+ T-cells (38). The positive prognostic impact of high CD8+ TIL density has already been demonstrated in ASCC $(9,11-13)$.

Previous studies have reported strong association of leukocytosis with peripheral blood neutrophilia in ASCC (23) and cervical cancer $(18,20,39)$. The impact of leukocytosis on DFS and OS in our study was mainly due to distant metastases, and experimental models showed that neutrophils play a major role in early metastatic spread (40). Tumors promote neutrophil release via G-CSF and CXCR2 ligands $(41,42)$ that can lead to chemo- and radioresistance $(39,43)$. Of note, we failed to identify a significant correlation between total peripheral WBC 

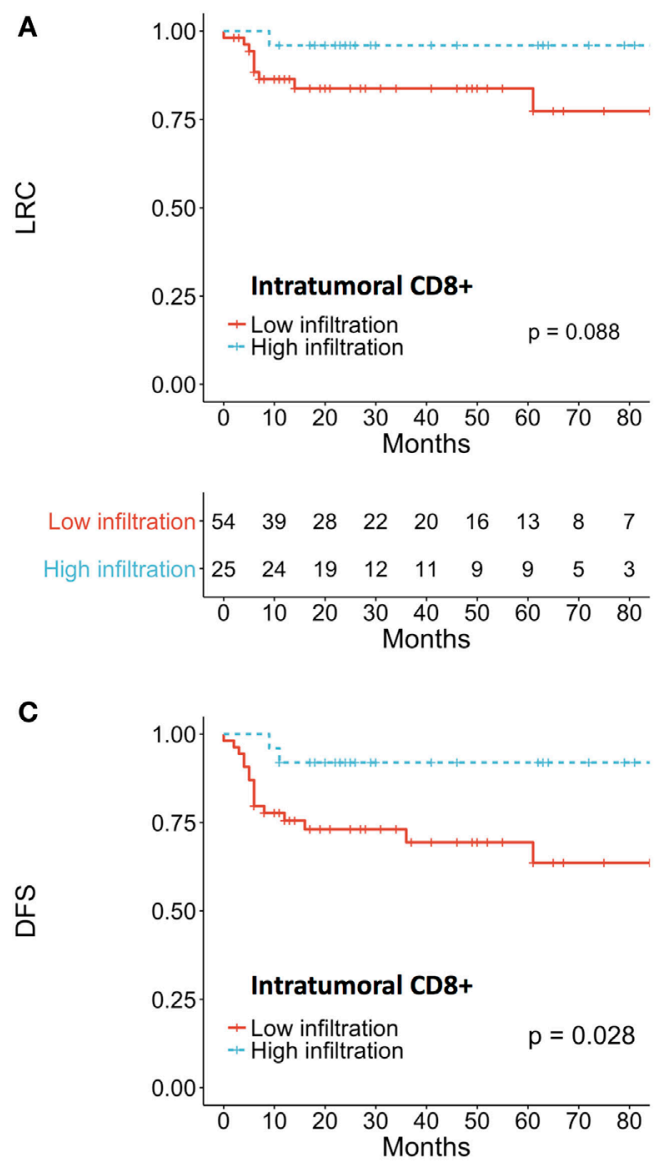

Low infiltration \begin{tabular}{ccccccccc}
54 & 39 & 27 & 22 & 18 & 15 & 12 & 8 & 7 \\
25 & 24 & 19 & 12 & 11 & 9 & 9 & 5 & 3 \\
\hline 0 & 10 & 20 & 30 & 40 & 50 & 60 & 70 & 80 \\
Months & & & &
\end{tabular}

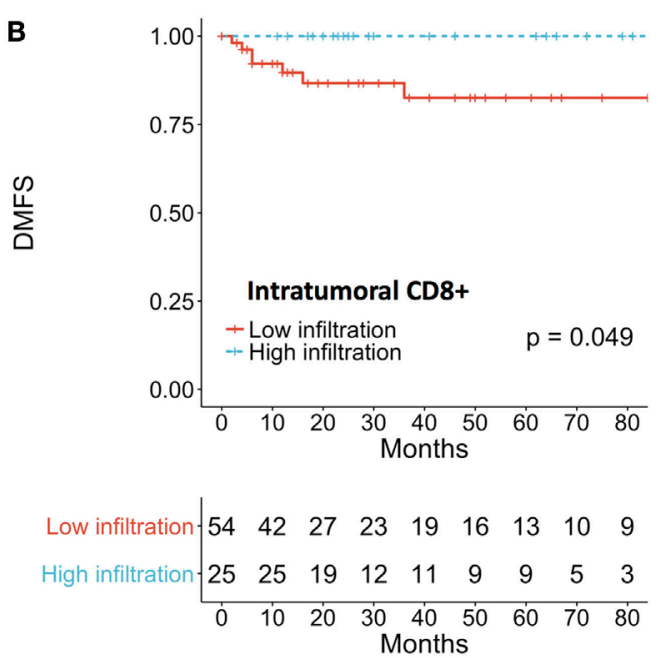

D

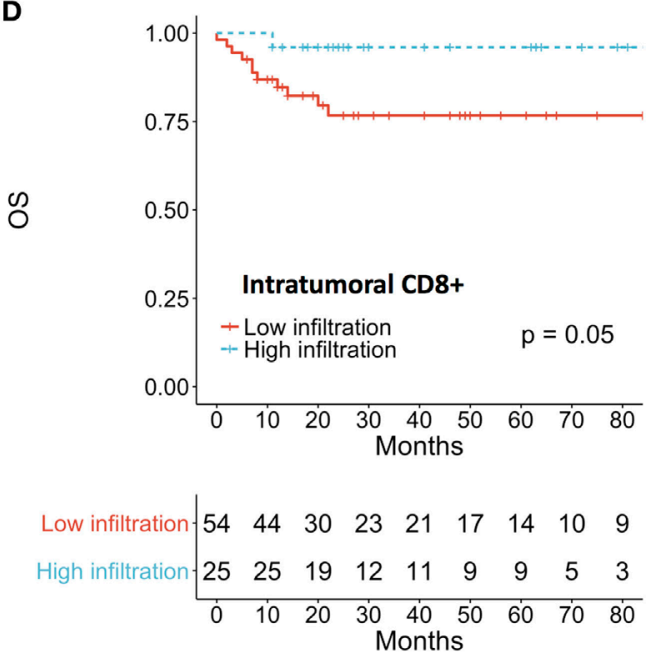

FIGURE 4 | Prognostic impact of intratumoral CD8+ T-cells on (A) LRC, (B) DMFS, (C) DFS, (D) OS. Patients were dichotomized according to the median intratumoral score. LRC, locoregional control; DMFS, distant-metastasis-free survival; DFS, disease-free survival; OS, overall survival.

and intratumoral neutrophils, which could be attributed to the fact that WBC count incorporates various peripheral blood cell populations.

The negative impact of elevated platelets on DMFS is in line with preclinical evidence that suggests an important role for platelets in metastasis (26). Similar data have been reported before in other tumor types, such as colorectal cancer $(27,28)$. Also, prophylactic aspirin that inhibits platelet activation has been shown to decrease metastatic incidence and cancer mortality (44).

We did not observe any significant prognostic influence of TAN on treatment outcome. Of note, a high variance has been reported regarding the prognostic value of neutrophils (45-50). One possible explanation for these discrepancies in literature could be the heterogeneous treatments and cohorts, and the use of different markers to evaluate TAN (CD11b, $\mathrm{CD} 15, \mathrm{MPO})$. Another explanation could be different compositions of the TME between malignancies. In cervical cancer, a negative prognostic impact of TAN is reported (51), whereas in squamous cell carcinoma of the head and neck, studies have reported positive and negative prognostic value $(52,53)$. Several approaches for targeting TAN are under clinical evaluation, for example, by blocking CXCR2 using the inhibitor reparixin (54). Interestingly, preclinical evidence has also suggested a synergistic effect of combining CXCR2 blockade with immune checkpoint inhibition (55).

Our study has several limitations. First, differential baseline blood count values, including neutrophils and lymphocytes, were unavailable in a large proportion of patients. Second, the retrospective design could have resulted in selection bias. Third, although our median follow-up of 26 months was relatively long, randomized trials have been reported with longer follow-up time. Fourth, immunohistochemical staining scoring was performed using a non-automated system due to the lack of international consensus and pathology standardization that constitutes another 
limitation of our work. These results clearly need to be validated in other cohorts, preferably in a prospective manner. In that context, we and others have previously investigated additional potential biomarkers relevant to the immune microenvironment, such as EGFR, surviving, Plk3, FOXP3, and PD-1/PD-L1 $(9,56-60)$. These could be potentially exploited in the future to generate robust prognostic models with several biomarkers to guide treatment stratification for future trials.

In conclusion, we here demonstrate that peripheral leukocytosis was associated with adverse clinical outcome in patients with ASCC treated with definitive CRT. This is the first study to show an inverse correlation between peripheral blood leukocytosis and intratumoral CD8+ TIL as well as between peritumoral MPO+ TAN and stromal CD8+ TIL that provide important mechanistic insight on the adverse impact of peripheral leukocytosis and the interplay between different immune cell populations in ASCC. These findings add to the previous literature on the role of leukocytosis and immune contexture in mediating response to treatment in ASCC and should lead to further investigations into the immune microenvironment of this disease.

\section{ETHICS STATEMENT}

This study was carried out in accordance with the recommendations of the ethics committee of the University Hospital Frankfurt am Main and approval from the institutional review board. All patients gave written informed consent in accordance with the Declaration of Helsinki.

\section{REFERENCES}

1. Jemal A, Simard EP, Dorell C, Noone A-M, Markowitz LE, Kohler B, et al. Annual Report to the Nation on the Status of Cancer, 1975-2009, featuring the burden and trends in human papillomavirus (HPV)-associated cancers and HPV vaccination coverage levels. J Natl Cancer Inst (2013) 105:175-201. doi:10.1093/jnci/djs491

2. James RD, Glynne-Jones R, Meadows HM, Cunningham D, Myint AS, Saunders MP, et al. Mitomycin or cisplatin chemoradiation with or without maintenance chemotherapy for treatment of squamous-cell carcinoma of the anus (ACT II): a randomised, phase 3, open-label, $2 \times 2$ factorial trial. Lancet Oncol (2013) 14:516-24. doi:10.1016/S1470-2045(13)70086-X

3. Gunderson LL, Winter KA, Ajani JA, Pedersen JE, Moughan J, Benson AB, et al. Long-term update of US GI intergroup RTOG 98-11 phase III trial for anal carcinoma: survival, relapse, and colostomy failure with concurrent chemoradiation involving fluorouracil/mitomycin versus fluorouracil/cisplatin. J Clin Oncol (2012) 30:4344-51. doi:10.1200/JCO.2012.43.8085

4. Ajani JA, Winter KA, Gunderson LL, Pedersen J, Benson AB, Thomas CR, et al. Prognostic factors derived from a prospective database dictate clinical biology of anal cancer: the intergroup trial (RTOG 98-11). Cancer (2010) 116:4007-13. doi:10.1002/cncr.25188

5. Gunderson LL, Moughan J, Ajani JA, Pedersen JE, Winter KA, Benson AB, et al. Anal carcinoma: impact of TN category of disease on survival, disease relapse, and colostomy failure in US Gastrointestinal Intergroup RTOG 98-11 phase 3 trial. Int J Radiat Oncol Biol Phys (2013) 87:638-45. doi:10.1016/ j.ijrobp.2013.07.035

6. De Vuyst H, Clifford GM, Nascimento MC, Madeleine MM, Franceschi S. Prevalence and type distribution of human papillomavirus in carcinoma and intraepithelial neoplasia of the vulva, vagina and anus: a meta-analysis. Int J Cancer (2009) 124:1626-36. doi:10.1002/ijc.24116

\section{AUTHOR CONTRIBUTIONS}

All authors designed and drafted the project. Clinical data collection and scoring of histochemical staining was done by DM, FR, PB, CR, and EF; RW performed pathological evaluation. All authors contributed to statistical analyses and $\mathrm{DM}, \mathrm{PB}$, and $\mathrm{EF}$ drafted the final manuscript with support from all co-authors. All authors performed final evaluation and agreed to be accountable for the content of the study.

\section{ACKNOWLEDGMENTS}

We are grateful to the excellent technical assistance of Mrs. Yvonne Michel, Senckenberg Institute of Pathology, Goethe-University, Frankfurt am Main, and Mr. Julius Oppermann, Department of Radiotherapy and Oncology, Goethe-University, Frankfurt am Main.

\section{SUPPLEMENTARY MATERIAL}

The Supplementary Material for this article can be found online at http://journal.frontiersin.org/article/10.3389/fimmu. 2017.01225/full\#supplementary-material.

FIGURE S1 | Association of pretreatment platelet count with (A) intratumoral and (B) stromal CD8+ T-cells.

FIGURE S2 | Prognostic impact of WBC on (A) LRC, (B) DMFS, (C) DFS, (D) OS. Patients were dichotomized according to a WBC count $\geq 10 \mathrm{nl}^{-1}$. LRC, locoregional control; DMFS, distant-metastasis-free survival; DFS, disease-free survival; OS, overall survival.

7. Serup-HansenE,LinnemannD, Skovrider-RuminskiW,HøgdallE, Geertsen PF Havsteen H. Human papillomavirus genotyping and p16 expression as prognostic factors for patients with American Joint Committee on Cancer stages I to III carcinoma of the anal canal. J Clin Oncol (2014) 32:1812-7. doi:10.1200/ JCO.2013.52.3464

8. Rödel F, Wieland U, Fraunholz I, Kitz J, Rave-Fränk M, Wolff HA, et al. Human papillomavirus DNA load and p16 ${ }^{\text {INK4a }}$ expression predict for local control in patients with anal squamous cell carcinoma treated with chemoradiotherapy: HPV16 DNA and p16 $6^{\text {INK4a }}$ Expression. Int J Cancer (2014) 136:278-88. doi:10.1002/ijc.28979

9. Balermpas P, Martin D, Wieland U, Rave-Fränk M, Strebhardt K, Rödel C, et al. Human papilloma virus load and PD-1/PD-L1, CD8+ and FOXP3 in anal cancer patients treated with chemoradiotherapy: rationale for immunotherapy. Oncoimmunology (2017) 6:e1288331. doi:10.1080/21624 02X.2017.1288331

10. Gooden MJM, de Bock GH, Leffers N, Daemen T, Nijman HW. The prognostic influence of tumour-infiltrating lymphocytes in cancer: a systematic review with meta-analysis. Br J Cancer (2011) 105:93-103. doi:10.1038/ bjc. 2011.189

11. Rubio CA, Nilsson PJ, Petersson F, Höög A, Blegen H, Chetty R. The clinical significance of massive intratumoral lymphocytosis in squamous cell carcinoma of the anus. Int J Clin Exp Pathol (2008) 1:376.

12. Gilbert DC, Serup-Hansen E, Linnemann D, Høgdall E, Bailey C, Summers J, et al. Tumour-infiltrating lymphocyte scores effectively stratify outcomes over and above p16 post chemo-radiotherapy in anal cancer. Br J Cancer (2016) 114:134-7. doi:10.1038/bjc.2015.448

13. Hu W-H, Miyai K, Cajas-Monson LC, Luo L, Liu L, Ramamoorthy SL. Tumor-infiltrating CD8(+) T lymphocytes associated with clinical outcome in anal squamous cell carcinoma. J Surg Oncol (2015) 112:421-6. doi:10.1002/ jso. 23998 
14. Fridman WH, Pagès F, Sautès-Fridman C, Galon J. The immune contexture in human tumours: impact on clinical outcome. Nat Rev Cancer (2012) 12:298-306. doi:10.1038/nrc3245

15. Murdoch C, Muthana M, Coffelt SB, Lewis CE. The role of myeloid cells in the promotion of tumour angiogenesis. Nat Rev Cancer (2008) 8:618-31. doi: $10.1038 / \mathrm{nrc} 2444$

16. Coffelt SB, Kersten K, Doornebal CW, Weiden J, Vrijland K, Hau C-S, et al. IL-17-producing $\gamma \delta \mathrm{T}$ cells and neutrophils conspire to promote breast cancer metastasis. Nature (2015) 522:345-8. doi:10.1038/nature14282

17. Coffelt SB, Wellenstein MD, de Visser KE. Neutrophils in cancer: neutral no more. Nat Rev Cancer (2016) 16:431-46. doi:10.1038/nrc.2016.52

18. Mabuchi S, Matsumoto Y, Isohashi F, Yoshioka Y, Ohashi H, Morii E, et al. Pretreatment leukocytosis is an indicator of poor prognosis in patients with cervical cancer. Gynecol Oncol (2011) 122:25-32. doi:10.1016/ j.ygyno.2011.03.037

19. Qiu M, Yuan Z, Luo H, Ruan D, Wang Z, Wang F, et al. Impact of pretreatment hematologic profile on survival of colorectal cancer patients. Tumour Biol (2010) 31:255-60. doi:10.1007/s13277-010-0024-x

20. Escande A, Haie-Meder C, Maroun P, Gouy S, Mazeron R, Leroy T, et al. Neutrophilia in locally advanced cervical cancer: a novel biomarker for imageguided adaptive brachytherapy? Oncotarget (2016) 7:74886-94. doi:10.18632/ oncotarget.12440

21. Banerjee R, Roxin G, Eliasziw M, Joseph K, Maclean A, Buie WD, et al. The prognostic significance of pretreatment leukocytosis in patients with anal cancer treated with radical chemoradiotherapy or radiotherapy. Dis Colon Rectum (2013) 56:1036-42. doi:10.1097/DCR.0b013e31829ab0d4

22. Toh E, Wilson J, Sebag-Montefiore D, Botterill I. Neutrophil:lymphocyte ratio as a simple and novel biomarker for prediction of locoregional recurrence after chemoradiotherapy for squamous cell carcinoma of the anus. Colorectal Dis (2014) 16:O90-7. doi:10.1111/codi.12467

23. Schernberg A, Escande A, Rivin Del Campo E, Ducreux M, Nguyen F, Goere D, et al. Leukocytosis and neutrophilia predicts outcome in anal cancer. Radiother Oncol (2017) 122:137-45. doi:10.1016/j.radonc.2016.12.009

24. Glynne-Jones R, Sebag-Montefiore D, Adams R, Gollins S, Harrison M, Meadows HM, et al. Prognostic factors for recurrence and survival in anal cancer: generating hypotheses from the mature outcomes of the first United Kingdom Coordinating Committee on Cancer Research Anal Cancer Trial (ACT I). Cancer (2013) 119:748-55. doi:10.1002/cncr.27825

25. Franco AT, Corken A, Ware J. Platelets at the interface of thrombosis, inflammation, and cancer. Blood (2015) 126:582-8. doi:10.1182/blood-2014-08531582

26. Gay LJ, Felding-Habermann B. Contribution of platelets to tumour metastasis. Nat Rev Cancer (2011) 11:123-34. doi:10.1038/nrc3004

27. Kozak MM, von Eyben R, Pai JS, Anderson EM, Welton ML, Shelton AA, et al. The prognostic significance of pretreatment hematologic parameters in patients undergoing resection for colorectal cancer. Am J Clin Oncol (2017) 40:405-12. doi:10.1097/COC.0000000000000183

28. Gu D, Szallasi A. Thrombocytosis portends adverse prognosis in colorectal cancer: a meta-analysis of 5,619 patients in 16 individual studies. Anticancer Res (2017) 37:4717-26. doi:10.21873/anticanres.11878

29. Dahlin AM, Henriksson ML, Van Guelpen B, Stenling R, Öberg Å, Rutegård J, et al. Colorectal cancer prognosis depends on T-cell infiltration and molecular characteristics of the tumor. Mod Pathol (2011) 24:671-82. doi:10.1038/ modpathol.2010.234

30. Denkert C, Loibl S, Noske A, Roller M, Müller BM, Komor M, et al. Tumor-associated lymphocytes as an independent predictor of response to neoadjuvant chemotherapy in breast cancer. J Clin Oncol (2010) 28:105-13. doi:10.1200/JCO.2009.23.7370

31. Peduzzi P, Concato J, Kemper E, Holford TR, Feinstein AR. A simulation study of the number of events per variable in logistic regression analysis. J Clin Epidemiol (1996) 49:1373-9. doi:10.1016/S0895-4356(96)00236-3

32. R Development Core Team. R: A Language and Environment for Statistical Computing. Vienna: R Foundation for Statistical Computing (2008). Available from: http://www.r-project.org

33. Powell DR, Huttenlocher A. Neutrophils in the tumor microenvironment. Trends Immunol (2016) 37:41-52. doi:10.1016/j.it.2015.11.008

34. Coussens LM, Zitvogel L, Palucka AK. Neutralizing tumor-promoting chronic inflammation: a magic bullet? Science (2013) 339:286-91. doi:10.1126/ science. 1232227
35. Rodriguez PC, Zea AH, DeSalvo J, Culotta KS, Zabaleta J, Quiceno DG, et al. L-arginine consumption by macrophages modulates the expression of CD3 zeta chain in T lymphocytes. J Immunol (2003) 171:1232-9. doi:10.4049/ jimmunol.171.3.1232

36. SippelTR, WhiteJ,NagK, Tsvankin V,Klaassen M,Kleinschmidt-DeMastersBK, et al. Neutrophil degranulation and immunosuppression in patients with GBM: restoration of cellular immune function by targeting arginase I. Clin Cancer Res (2011) 17:6992-7002. doi:10.1158/1078-0432.CCR-11-1107

37. Molon B, Ugel S, Del Pozzo F, Soldani C, Zilio S, Avella D, et al. Chemokine nitration prevents intratumoral infiltration of antigen-specific T cells. J Exp Med (2011) 208:1949-62. doi:10.1084/jem.20101956

38. Pang Y, Gara SK, Achyut BR, Li Z, Yan HH, Day C-P, et al. TGF- $\beta$ signaling in myeloid cells is required for tumor metastasis. Cancer Discov (2013) 3:936-51. doi:10.1158/2159-8290.CD-12-0527

39. Mabuchi S, Matsumoto Y, Kawano M, Minami K, Seo Y, Sasano T, et al. Uterine cervical cancer displaying tumor-related leukocytosis: a distinct clinical entity with radioresistant feature. J Natl Cancer Inst (2014) 106:7. doi:10.1093/jnci/ dju147

40. Wculek SK, Malanchi I. Neutrophils support lung colonization of metastasis-initiating breast cancer cells. Nature (2015) 528:413-7. doi:10.1038/ nature 16140

41. Blaisdell A, Crequer A, Columbus D, Daikoku T, Mittal K, Dey SK, et al. Neutrophils oppose uterine epithelial carcinogenesis via debridement of hypoxic tumor cells. Cancer Cell (2015) 28:785-99. doi:10.1016/ j.ccell.2015.11.005

42. Casbon A-J, Reynaud D, Park C, Khuc E, Gan DD, Schepers K, et al. Invasive breast cancer reprograms early myeloid differentiation in the bone marrow to generate immunosuppressive neutrophils. Proc Natl Acad Sci U S A (2015) 112:E566-75. doi:10.1073/pnas.1424927112

43. Kawano M, Mabuchi S, Matsumoto Y, Sasano T, Takahashi R, Kuroda H, et al. The significance of G-CSF expression and myeloid-derived suppressor cells in the chemoresistance of uterine cervical cancer. Sci Rep (2015) 5:18217. doi:10.1038/srep18217

44. Rothwell PM, Fowkes FGR, Belch JFF, Ogawa H, Warlow CP, Meade TW. Effect of daily aspirin on long-term risk of death due to cancer: analysis of individual patient data from randomised trials. Lancet Lond Engl (2011) 377:31-41. doi:10.1016/S0140-6736(10)62110-1

45. Caruso RA, Bellocco R, Pagano M, Bertoli G, Rigoli L, Inferrera C. Prognostic value of intratumoral neutrophils in advanced gastric carcinoma in a highrisk area in northern Italy. Mod Pathol (2002) 15:831-7. doi:10.1097/01. MP.0000020391.98998.6B

46. Jensen HK, Donskov F, Marcussen N, Nordsmark M, Lundbeck F, von der Maase $\mathrm{H}$. Presence of intratumoral neutrophils is an independent prognostic factor in localized renal cell carcinoma. J Clin Oncol (2009) 27:4709-17. doi:10.1200/JCO.2008.18.9498

47. Jensen TO, Schmidt H, Møller HJ, Donskov F, Høyer M, Sjoegren P, et al. Intratumoral neutrophils and plasmacytoid dendritic cells indicate poor prognosis and are associated with pSTAT3 expression in AJCC stage I/II melanoma. Cancer (2012) 118:2476-85. doi:10.1002/cncr.26511

48. Rao H-L, Chen J-W, Li M, Xiao Y-B, Fu J, Zeng Y-X, et al. Increased intratumoral neutrophil in colorectal carcinomas correlates closely with malignant phenotype and predicts patients' adverse prognosis. PLoS One (2012) 7:e30806. doi:10.1371/journal.pone.0030806

49. Droeser RA, Hirt C, Eppenberger-Castori S, Zlobec I, Viehl CT, Frey DM, et al. High myeloperoxidase positive cell infiltration in colorectal cancer is an independent favorable prognostic factor. PLoS One (2013) 8:e64814. doi:10.1371/journal.pone.0064814

50. Zhou L, Xu L, Chen L, Fu Q, Liu Z, Chang Y, et al. Tumor-infiltrating neutrophils predict benefit from adjuvant chemotherapy in patients with muscle invasive bladder cancer. Oncoimmunology (2017) 6:e1293211. doi:10.1080/ 2162402X.2017.1293211

51. Matsumoto Y, Mabuchi S, Kozasa K, Kuroda H, Sasano T, Yokoi E, et al. The significance of tumor-associated neutrophil density in uterine cervical cancer treated with definitive radiotherapy. Gynecol Oncol (2017) 145:469-75. doi:10.1016/j.ygyno.2017.02.009

52. Millrud CR, Kågedal Å, Kumlien Georén S, Winqvist O, Uddman R, Razavi R, et al. NET-producing CD16(high) CD62L(dim) neutrophils migrate to tumor sites and predict improved survival in patients with HNSCC. Int J Cancer (2017) 140:2557-67. doi:10.1002/ijc.30671 
53. Trellakis S, Bruderek K, Dumitru CA, Gholaman H, Gu X, Bankfalvi A, et al. Polymorphonuclear granulocytes in human head and neck cancer: enhanced inflammatory activity, modulation by cancer cells and expansion in advanced disease. Int J Cancer (2011) 129:2183-93. doi:10.1002/ijc.25892

54. Schott AF, Goldstein LJ, Cristofanilli M, Ruffini PA, McCanna S, Reuben JM, et al. Phase Ib pilot study to evaluate reparixin in combination with weekly paclitaxel in patients with HER-2-negative metastatic breast cancer. Clin Cancer Res (2017) 23:5358-65. doi:10.1158/1078-0432.CCR-16-2748

55. Highfill SL, Cui Y, Giles AJ, Smith JP, Zhang H, Morse E, et al. Disruption of CXCR2-mediated MDSC tumor trafficking enhances anti-PD1 efficacy. Sci Transl Med (2014) 6:237ra67. doi:10.1126/scitranslmed.3007974

56. Rödel F, Martin D, Helmke C, Balermpas P, Fokas E, Wieland U, et al. Polo like kinase 3 and phosphoT273 caspase- 8 are associated with improved local tumor control and survival in patients with anal carcinoma treated with concomitant chemoradiotherapy. Oncotarget (2016) 7:53339-49. doi:10.18632/ oncotarget.10801

57. Fraunholz I, Rödel C, Distel L, Rave-Fränk M, Kohler D, Falk S, et al. High survivin expression as a risk factor in patients with anal carcinoma treated with concurrent chemoradiotherapy. Radiat Oncol (2012) 7:1. doi:10.1186/1748-717X-7-88

58. Gilbert DC, Williams A, Allan K, Stokoe J, Jackson T, Linsdall S, et al p16INK4A, p53, EGFR expression and KRAS mutation status in squamous cell cancers of the anus: correlation with outcomes following chemo-radiotherapy. Radiother Oncol (2013) 109:146-51. doi:10.1016/j.radonc.2013.08.002

59. Martin V, Zanellato E, Franzetti-Pellanda A, Molinari F, Movilia A, Paganotti A, et al. EGFR, KRAS, BRAF, and PIK3CA characterization in squamous cell anal cancer. Histol Histopathol (2014) 29:513-21. doi:10.14670/HH29.10 .513

60. Fraunholz I, Rödel F, Kohler D, Diallo-Georgiopoulou M, Distel L, Falk S, et al. Epidermal growth factor receptor expression as prognostic marker in patients with anal carcinoma treated with concurrent chemoradiation therapy. Int J Radiat Oncol Biol Phys (2013) 86:901-7. doi:10.1016/j.ijrobp.2013.03.039

Conflict of Interest Statement: The authors declare that the research was conducted in the absence of any commercial or financial relationships that could be construed as a potential conflict of interest.

Copyright (c) 2017 Martin, Rödel, Winkelmann, Balermpas, Rödel and Fokas. This is an open-access article distributed under the terms of the Creative Commons Attribution License (CC BY). The use, distribution or reproduction in other forums is permitted, provided the original author(s) or licensor are credited and that the original publication in this journal is cited, in accordance with accepted academic practice. No use, distribution or reproduction is permitted which does not comply with these terms. 